\title{
On the robustness of DCT-based compression algorithms for space applications
}

\author{
S. Avramenko, M. Sonza Reorda, M. Violante \\ Politecnico di Torino, Torino, Italy
}

\begin{abstract}
High compression ratio is crucial to cope with the large amounts of data produced by telemetry sensors and the limited transmission bandwidth typical of space applications. A new generation of telemetry units is under development, based on Commercial Off-The-Shelf (COTS) components that may be subject to misbehaviors due to radiation-induced soft errors. The purpose of this paper is to study the impact of soft errors on different configurations of a discrete cosine transform (DCT)based compression algorithm. This work's main contribution lies in providing some design guidelines.
\end{abstract}

Keywords-Soft Errors; Lossy Compression Algorithm; Discrete Cosine Transform; Reliability; Register-level Fault Injection; Commercial Off-The-Shelf;

\section{INTRODUCTION}

In space applications (both for satellites and for launchers), systems dedicated to the compression of data are mandatory, since bandwidth and storage are limited. Although some studies has been done [1], the research effort is required to develop suitable and flexible compression algorithms, able to match all the above constraints. Reliability is an important design constraint for space applications. To face this issue, the traditional approach was based on using space qualified hardware. Commercial Off-The-Shelf components (COTS) offer reduction in weight and energy consumption, and higher computational power compared to rad-hard counterparts, while poses challenges about radiation effects mitigation [2]. The European Commission decided to fund the MaMMoTH-Up project, whose main target is to develop a new generation of telemetry systems for Ariane launchers, based on COTS components. In this paper, the relationship between the different parameters of the considered DCT-based algorithm and the robustness has been investigated. The main contribution of this paper lies in presenting some guidelines to be used by the system architect. The results related to the lossless compression algorithms analyzed in [3] are compared with the figures related to the lossy algorithm analyzed in this paper. The paper is organized in the following manner. An overview of the considered compression algorithm is given in Section II. Section III describes the Fault Injection environment. Section IV reports the experimental results and the related considerations. The comparison with the results of the previous work is presented in this section. Section V draws some conclusions.

\author{
G. Fey, J.-G. Mess, R. Schmidt \\ German Aerospace Center (DLR), Bremen, Germany
}

\section{BACKGROUND ON CONSIDERED DCT-BASED COMPRESSION ALGORITHM}

The proposed algorithm uses discrete cosine transforms in combination with pre- and post-processing steps for lossy compression of one-dimensional time series data. The discrete cosine transform (DCT) is an orthogonal transform [4] is applied to (pre-processed) fixed length blocks of data, first few coefficients are then retained, which are large compared to higher order ones. The length of the block of data to be separately converted by one-dimensional DCT, Width, as a parameter of the algorithm; two different values of Width: 8 elements per block and 16 elements per block, has been considered. The one-dimensional DCT is used to achieve twodimensional DCT [5] on data block of dimension Width* Width. Before the DCT is applied all the data is scaled by Pre-factor (pf). The post-processing consists in a thin out of the DCT coefficients, which are the result of compression, by means of a threshold value Threshold and the limit to the retained coefficients Coeff_Num. Finally, the algorithm stores the positions of discarded coefficients as a sparse bit string, resulting in a negligible overhead.

\section{THE EXPERIMENTAL SETUP}

The overall experimental environment is composed of two subenvironments: a run-time environment and a fault injection environment.

\section{A. The run-time environment}

Since the OpenRISC architecture is intended for the final deployment, the run-time environment is based on its Instruction Set Simulator (ISS). It is in charge of executing the DCT compression algorithm on representative set of values coming from sensors. The software stack has been designed to be executed in bare metal.

\section{$B$. The fault injection environment}

Since the effects of SEUs was under investigation, the fault injection environment has been developed accordingly. Single bit flips inside the processor's general purpose registers were considered [6]. Each fault to be injected is generated by randomly choosing the target register, the bit of that register, and the time the fault is injected. Faults are classified according to the following categories: 
- Silent: the fault had no effect.

- Wrong output: the fault corrupts the output but does not affect execution time.

- Detected: the fault triggers some hardware exception:

- Timeout error: execution time is affected.

- Bus error: invalid memory address access.

- Alignment error: misaligned memory address access.

\section{EXPERIMENTAL RESULTS}

The experiments have been performed on the OpenRISC ISS, executing the DCT-based compression algorithm with different parameter sets, as reported in Table I. One input data set has been considered: pressure sensor, with 8 bits per sample. A Montecarlo-based approach has been used to validate the statistic meaningfulness of the data. A set of 100,000 faults has been injected. In this paper, the compression ratio is defined as the ratio between the size of original data and compressed data.

TABLE I. PARAMETER SETS DETAILS

\begin{tabular}{|l|r|r|r|r|}
\hline & \multicolumn{1}{|c|}{ Width } & Coeff_Num & Pre-factor & Threshold \\
\hline Par. set 01 & 8 & 56 & 0.125 & 0.5 \\
\hline Par. set 02 & 8 & 48 & 1.0 & 4.5 \\
\hline Par. set 03 & 8 & 16 & 2.0 & 1.0 \\
\hline Par. set 04 & 16 & 64 & 2.0 & 1.0 \\
\hline
\end{tabular}

TABLE II. RESULTS FOR DIFFERENT PARAMETER SETS

\begin{tabular}{|l|r|r|r|r|}
\hline & Par. set 01 & Par. set 02 & Par. set 03 & Par. set 04 \\
\hline & {$[\%]$} & {$[\%]$} & {$[\%]$} & {$[\%]$} \\
\hline Compression Ratio & 391 & 690 & 750 & 9,569 \\
\hline \hline Wrong Output & 13.4 & 12.0 & 11.8 & 4.8 \\
\hline Silent & 79.3 & 80.3 & 80.5 & 86.3 \\
\hline Detected & 7.3 & 7.7 & 7.7 & 8.9 \\
\hline
\end{tabular}

Table II allows the following observations:

- Considering the wrong output as a metric of robustness, it is possible to observe that the robustness increases as compression ratio increases.

- The detected percentage, it is almost insensitive to all the parameters but Width, while still some relationship with Pre-factor parameter exists. Indeed, Par. set 04, with width 16 , has the highest rate of detection. This can be explained because the Width parameter defines the size of data to be considered for a conversion. Thus, it deeply affects the number of operations per data sample performed.

The subcategories of Detected are shown in Table III. Since it has been observed that Width is the most relevant parameter for the detection mechanism activation, Par. set 01 and Par. set 02 has not been considered. From Table III, detection is triggered mainly by both timeout error and bus error.

Table IV reports the results achieved for the considered DCTbased lossy compression algorithm, with the results achieved for the lossless LZW12 and RICE algorithms [3]. This table only present figures related to Par. set 03 and Par. set 04, in order to consider a parameter set for both values of Width. Experiments from [3] have been repeated on the same data sets used in this paper.

TABLE III. SUBCATEGORIES OF DETECTED GROUP

\begin{tabular}{|l|r|r|}
\hline & Par. set 03 & Par. set 04 \\
\hline & {$[\%]$} & {$[\%]$} \\
\hline Timeout error & 2.3 & 2.9 \\
\hline Bus error & 4.7 & 5.2 \\
\hline Alignment error & 0.7 & 0.8 \\
\hline \hline TOTAL (Detected) & 7.7 & 8.9 \\
\hline
\end{tabular}

TABLE IV. RESULTS FOR LOSSY AND LOSSLESS ALGORITHMS

\begin{tabular}{|l|r|r|r|r|}
\hline & Par. set 03 & Par. set 04 & RICE & \multicolumn{1}{|c|}{ LZW12 } \\
\hline & {$[\%]$} & {$[\%]$} & {$[\%]$} & {$[\%]$} \\
\hline Compression Ratio & 750 & 9,569 & 2,552 & 1,225 \\
\hline \hline Wrong Output & 11.8 & 4.8 & 24.7 & 5.3 \\
\hline Silent & 80.5 & 86.3 & 51.4 & 80.5 \\
\hline Detected & 7.7 & 8.9 & 23.9 & 14.2 \\
\hline
\end{tabular}

From the figures of the Table IV, the considered DCT-based algorithm is qualitatively better than the lossless ones.

\section{CONCLUSIONS}

This paper investigates the relationship between the parameters of a DCT-based algorithm and the robustness against SEU faults. The work of this paper provides information to allow an early the reliability analysis, for what it concerns SEUs. The experimental results highlight some relationship between DCTbased algorithm's parameters and the effects of SEUs in general purpose registers. Experimental results show that the robustness increases as compression ratio increases. The future work will target the validation of the considerations made in this work, by means of RTL fault injection. It will also explore the possibility of improving dependability of the algorithm.

\section{ACKNOWLEDGEMENTS}

This work has been supported by the European Commission through the Horizon 2020 Project No. 637616 (MaMMoTHUP).

\section{REFERENCES}

[1] A. G. Schmidt, M. French, "Fast lossless image compression with Radiation Hardening by hardware/software co-design on platform FPGAs", Application-Specific Systems, Architectures and Processors (ASAP), 2013 IEEE 24th International Conference, pp. 103 - 106.

[2] O. Goloubeva, M. Rebaudengo, M. Sonza Reorda and M. Violante, Software-implemented Hardware Fault Tolerance, 2006, Springer.

[3] S. Avramenko, M. Sonza Reorda, M. Violante, G. Fey, "Analysis of the Effects of Soft Errors on Compression Algorithms Through Fault Injection Inside Program Variables", IEEE Latin-American Test Symposium (LATS).

[4] N. Ahmed, T. Natarajan, and K. R. Rao, "Discrete Cosine Transfom," IEEE Transactions on Computers, vol. 23, no. 1, pp. 90-93, Jan. 1974.

[5] Y.-M. Huang and J.-L. Wu, "A Refined Fast 2-D Discrete Cosine Transform Algorithm,” IEEE Transactions on Signal Processing, vol. 47, no. 3, pp. 904-907, Mar. 1999.

[6] M-C. Hsueh, T. K. Tsai, R. K. Iyer, "Fault Injection Techniques and Tools", IEEE Computer, Vol. 30, No. 4, April 1997, pp. 75-82. 